\title{
Pickup development for short low-charge bunches in $\mathrm{x}$-ray free-electron lasers
}

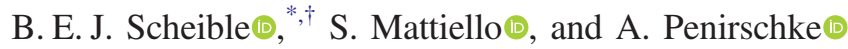 \\ Technische Hochschule Mittelhessen, Wilhelm-Leuschner-Straße 13, 61169 Friedberg, Germany \\ M. K. Czwalinna® and H. Schlarbఠ \\ Deutsches Elektronen-Synchrotron DESY, Notkestraße 85, 22607 Hamburg, Germany \\ W. Ackermann and H. De Gersem $\odot$ \\ Technische Universität Darmstadt, Schloßgartenstraße 8, 64289 Darmstadt, Germany
}

(Received 2 February 2021; accepted 1 June 2021; published 21 July 2021)

\begin{abstract}
The all-optical synchronization systems used in various x-ray free-electron lasers (XFEL) such as the European XFEL usually include bunch arrival-time monitors (BAM), which observe the transient fields of passing electron bunches through their pickups. The extracted signal is used to modulate the amplitude of a reference laser pulse in a Mach-Zehnder type electro-optical modulator. The laser pulse is typically much shorter than the voltage signal and interacts only with an instantaneous value of the applied signal. With the emerging demand for future experiments with short FEL shots, fs precision is required for the synchronization systems even with $1 \mathrm{pC}$ bunches. Since the sensitivity of the BAM depends in particular on the slope of the bipolar signal at the zero crossing and thus, also on the bunch charge, a redesign with the aim of a significant increase by optimized geometry and bandwidth is inevitable. In this contribution the theoretical foundations of the pickup signal are aggregated and treated with a focus on short bunches as well as a general formulation. The analytical treatment reveals design limitations and suggests several approaches for further development. Based on these considerations a new pickup concept with possible advantages in manufacturing and temporal resolution is simulated and its performance is compared to the previous concept. The design offers good potential and a significant improvement of slope and voltage is found. Nevertheless the target set for $1 \mathrm{pC}$ operation was not fully reached yet and further optimization is necessary. The observed improvement is mainly achieved by the reduced distance to the beam and a higher bandwidth.
\end{abstract}

DOI: 10.1103/PhysRevAccelBeams.24.072803

\section{INTRODUCTION}

Free-electron lasers (FELs) became an important light source for experiments in various fields since they provide short pulses with extreme brilliance in atomic length and time scales [1]. FELs are well suited for applications in pump-probe experiments [1], where the timing jitter is specifically critical [2], as well as for capturing image sequences with atomic resolution on fs timescales, even below the FEL repetition rate $[1,3,4]$.

*bernhard.scheible@iem.thm.de

Also at Technische Universität Darmstadt, Schloßgartenstraße 8, 64289 Darmstadt, Germany.

Published by the American Physical Society under the terms of the Creative Commons Attribution 4.0 International license. Further distribution of this work must maintain attribution to the author(s) and the published article's title, journal citation, and DOI.
For the generation of short x-ray pulses, FELs with short and low charge electron bunches $(\leq 1 \mathrm{pC})$ have been found as a favorable option $[5,6]$. Short bunches may shorten the $x$-ray pulse, reduce timing jitter and lead to single-spike operation, if sufficiently small compared to the cooperation length of the SASE process $[2,5,6]$. The European XFEL (EuXFEL) was upgraded from initially $1 \mathrm{nC}$ electron bunches to cover a range from 0.02 to $1 \mathrm{nC}$ [7] with a possible bunch length below $3 \mathrm{fs}$ in the undulator section [8]. Moreover, a decrease to bunch charges of $1 \mathrm{pC}$ is targeted.

The application in time-resolved experiments entails tight requirements for the overall machine synchronization in order to reduce the timing jitter [2]. The timing information is also used for postprocessing experimental data [1]. The synchronization concerns all critical subsystems, specifically in the injector and if present the seeding and the pump laser [1]. Furthermore, the instrumentation must be suited for a broad spectrum of operation modes with different bunch properties even in a single bunch train $[9,10]$. Besides, bunch arrival-time monitors 
(BAMs) are installed throughout the whole facility, thus experiencing different bunch properties.

A tremendous improvement in synchronization stability and BAM resolution, surpassing rf techniques, and a reduction of arrival time as well as energy jitter was achieved by the implementation of an all-optical synchronization system with two different feedback loops [11], which was developed at the Deutsches ElektronenSynchrotron (DESY). However, an rf-based synchronization scheme with resonant cavities is still in use and under continuous further development. Starting with the rf-synchronization scheme, some notable examples of both types are presented in the following paragraphs.

For the Pohang Accelerator Laboratory (PAL-XFEL) a resolution of 25 fs has been reported in 2016 for the type 1 and type 2 cavities combined with the low level rf [12]. These values were found in a 20 min measurement with $200 \mathrm{pC}$ bunches at the PAL-XFEL injector test facility [12]. In 2017 the authors of [13] claim a systematic error of $11.2 \mathrm{fs}$ for the phase cavity system implemented in the PAL-XFEL. At the Japanese SPring-8 Angstrom Compact Laser (SACLA) a temporal resolution of $41 \mathrm{fs}$ was found in a $10 \mathrm{~s}$ measurement with $200 \mathrm{pC}$ bunches utilizing two adjacent beam position monitors [14]. For the Linac Coherent Light Source (LCLS) at SLAC National Accelerator Laboratory 13 fs with $250 \mathrm{pC}$ charge in normal operation and $27 \mathrm{fs}$ with $20 \mathrm{pC}$ in short pulse mode have been reported as rms noise [15]. The Shanghai soft x-ray FEL facility (SXFEL), with comparably high bunch charges $(500 \mathrm{pC})$, targets a temporal resolution below $100 \mathrm{fs}$ [16]. The rf-type configuration was selected because it is less complex and expensive than the electro-optical scheme and suited to meet the required resolution [16]. A system comprising four dual-cavity BAMs was built to measure arrival time and time of flight [16]. Initially a measured short-term resolution of 67.7 fs with 200 pC bunches was reported for the dual-cavity BAMs [17]. In 2021 the authors of [16] claim to have reached a resolution of $13 \mathrm{fs}$ for the flight time and about $45 \mathrm{fs}$ for the arrival time in a 20 min measurement with $500 \mathrm{pC}$ bunches. Nonetheless to achieve a better resolution for the arrivaltime measurement it is foreseen to test an electro-optical synchronization scheme at the SXFEL as well [18].

In 2009 FERMI@ELETTRA became the first linacbased FEL to be fully synchronized by the electro-optical synchronization scheme developed at DESY [19]. A resolution below 8 fs was found for $500 \mathrm{pC}$ bunches [19]. The scheme was also implemented in the SwissFEL $\mathrm{x}$-ray free-electron laser. At the SwissFEL Injector Test Facility first the measured resolution was 10 to $13 \mathrm{fs}$ with 90 to $200 \mathrm{pC}$ bunches [20]. The authors of [20] expected an improvement for the scaled pickups, which were foreseen for implementation in the $16 \mathrm{~mm}$ SwissFEL beampipe. In accordance a resolution below $10 \mathrm{fs}$ was reported for 30 to 200 pC bunches with smaller beampipe diameter and improved electronics at the SwissFEL [21]. Recently the performance of the state-of-the-art system was evaluated at the EuXFEL as well. The correlation of two adjacent monitors with less than $1 \mathrm{~m}$ distance was analyzed. Examining the measured arrival times for a period of 1 min gave a timing jitter of approximately $6 \mathrm{fs}$ rms caused by the BAM resolution and critical parts of the reference laser distribution system [22].

In the scope of this work, we will cover the electrooptical synchronization scheme in the realization implemented at EuXFEL at DESY, where the basic scheme also remained unchanged despite some updates $[23,24]$.

In the following section the basic working principle and main components of the BAMs are introduced with special attention on the state-of-the-art cone-shaped pickups. The schematic BAM description is followed by an in-depth analytical discussion of the voltage signal, regarding different limiting cases as well as a general solution, to identify principal parameters determining the BAM resolution and their effect on the signal shape. For design purposes an additional numerical treatment is introduced. These considerations are the basis for three designs, which are presented at the end of this paper. At first a reference design is defined by reduction of the beampipe aperture, similar to the BAM structure implemented in the SwissFEL due to their facility parameters [20]. The second design is a $90 \mathrm{GHz}$ coneshaped pickup presented at the 8th International Beam Instrumentation Conference, IBIC2019 [25]. At last a BAM on a printed circuit board is introduced. While none of these designs could fully achieve the desired improvement they still offer potential for further optimization.

\section{ALL-OPTICAL SYNCHRONIZATION SYSTEM}

The all-optical synchronization system, as successfully tested at the Free-Electron Laser in Hamburg (FLASH) by Löhl [11], mainly comprises a mode-locked reference laser, length stabilized fiber links and different end stations for synchronization and arrival-time measurement $[10,11,23$, $24,26,27]$. The arrival time is nondestructively measured with respect to the reference laser in the BAM, which includes high-bandwidth pickup electrodes in the rf unit, a Mach-Zehnder type electro-optical modulator (EOM) and the data acquisition system (DAQ) [10,27].

\section{A. Basic working principle of BAM}

The transient electric fields of passing electron bunches are extracted in the rf unit and, if foreseen, initially processed with analog components like rf combiners, limiters or attenuators $[11,23]$. The received bipolar signal is transmitted via coaxial cables to the EOM [23], there it is probed at its zero crossing (ZC) by the reference laser [11]. Any temporal deviation will lead to an additional voltage, which the EOM turns into an amplitude modulation of the laser pulse. Therefore, the laser amplitude holds the timing 
information, which can be retrieved in the DAQ [11]. The signal slope at its zero crossing strongly influences the BAM's temporal resolution. The minimum design requirement for the currently installed BAM was $\geq 300 \mathrm{mV} \mathrm{ps}^{-1}$ with $20 \mathrm{pC}$ bunches [23].

\section{B. rf unit and pickups}

The rf unit so far comprises four identical pickups mounted circularly around the beam line. The original pickups used at FLASH were of button type and designed for a $10 \mathrm{GHz}$ bandwidth [28]. This pickup struggled with ringing and strong signal reflections at the alumina vacuum feedthrough [28] degrading the signal strength and resolution for charges below $150 \mathrm{pC}$ [29]. Hence, a new design was required [29].

A novel pickup (Fig. 1, left), similar to those designed for the CERN linear collider test facility [30], was proposed as a solution in FEL applications [31]. The cone-shaped design, finalized in [23], with $40 \mathrm{GHz}$ bandwidth became a new standard device used at the EuXFEL and at other FELs $[32,33]$.

Due to high losses in the rf path a design update was necessary (Fig. 1, right). The second generation of coneshaped pickups was optimized towards a maximum signal voltage at the cost of its slope by increasing the active surface and letting the cone slightly protrude into the beampipe [24]. A limit was reached when a larger surface, e.g., more than a few bunch lengths, decreases the slope without a further increase in peak-to-peak voltage [24]. The protrusion increases the inductance, which unfavorably deforms the signal shape [24]. Nonetheless, a combination of both modifications increased the peak-to-peak voltage sufficiently for $20 \mathrm{pC}$ bunches while maintaining an acceptable slope [24].

The rf front end also contains an rf-power combiner, which combines the signal of two opposite pickups to compensate for the orbit dependency [34]. The combiner is placed in the vicinity of the beam line in order to mitigate the influence of phase shifts and attenuation on the signal. Approximately $20 \mathrm{~cm}$ long coaxial cables were matched

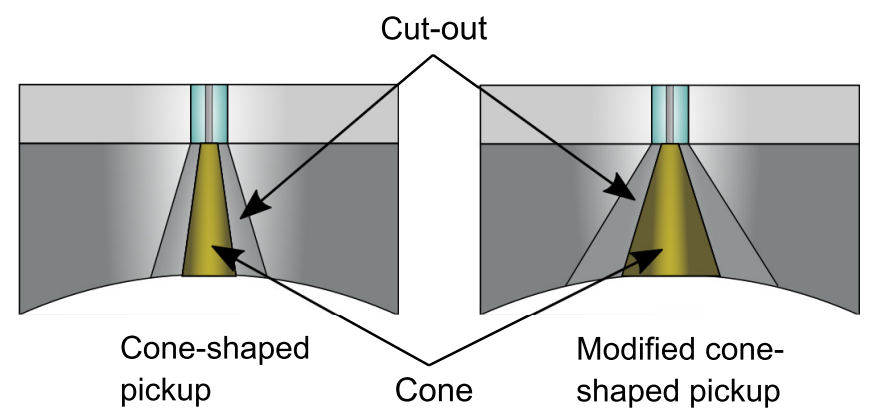

FIG. 1. Cross section of the first generation cone-shaped pickup (left) and the modified second generation cone-shaped pickup for high peak-to-peak voltage (right) adapted from [24]. and used for the transmission line connecting the two pickups with the combiner $[23,35]$.

Originally radiation hard silicon dioxide semirigid coaxial cables from Times Microwave have been proposed for the about $2 \mathrm{~m}$ long path from pickup to EOM [35]. Due to the semirigid behavior and high costs, it is worthwhile to consider the use of high-bandwidth low-loss Polytetrafluoroethylene (PTFE) cables. PTFE cables have a moderate price and may improve the sensitivity, but are less resistant to radiation damage [36]. An examination of different cables at the ELBE accelerator at HelmholtzZentrum Dresden-Rossendorf (HZDR) under realistic test conditions showed that the use of some PTFE cables is possible after careful evaluation [36]. Consistently the SwissFEL was equipped with PhaseMaster160 cables (Teledyne) [32] and the EuXFEL uses PhaseMaster160 cables as well, specifically absolute phase matched semirigid cables to connect two pickups with a combiner and hardarmored semiflex cables for the longer distance to the EOM.

\section{Electro-optical unit}

In the electro-optical unit an optical reference signal is shaped according to the rf signal applied to the EOM as described in the section on the basic working principle. The main components involved are the optical laser oscillator (OLO), the optical distribution and the EOM.

The reference signal is provided by the OLO locked to the $1.3 \mathrm{GHz}$ rf master oscillator [27]. At the EuXFEL a $1553 \mathrm{~nm}$ laser is used with a repetition rate of $216.7 \mathrm{MHz}$ giving a laser pulse every $4.6 \mathrm{~ns}$ [27]. The FWHM of these laser pulses is approximately 1 ps [10].

The pulses are distributed via polarization maintaining optical fibers [22], actively stabilized by the link stabilization units [37]. For this purpose the round-trip time of laser pulses is measured with balanced optical cross-correlators $[22,27]$. Changes of the optical length are compensated with piezo-based fiber stretchers and $4 \mathrm{~ns}$ long optical delay lines [22,27].

For generating a clock signal for the DAQ about $10 \%$ of the incoming reference pulses is split off, the remaining part is amplified and guided through a free-space optical delay stage with a range of \pm 130 ps to keep the reference pulses in perfect time overlap with the rf signals from the electron bunches inside of the EOM [38].

The key component of the electro-optic unit is the EOM. A commercial lithium niobate $\left(\mathrm{LiNbO}_{3}\right)$ Mach-Zehnder type EOM is used to modulate the amplitude of reference laser pulses. It needs to enable the rf signal's full $40 \mathrm{GHz}$ bandwidth. Additionally, it has to cope with the short, high energy laser pulses and must withstand the maximum voltage carried by the rf signal. This has to be considered specifically if the rf unit is designed to drive the EOMs with low-charge bunches, while the machine takes a wide range of charges. 


\section{Data acquisition system}

The DAQ electronics at DESY are built modularly using the open $\mu$ TCA standard [38]. It currently includes three $800 \mathrm{MHz}$ photodiodes, of which one is dedicated to receive the clock signal [38]. Both of the other diodes receive the modulated signal of one of the EOMs $[10,38]$. The electrical output with FWHM of about $1 \mathrm{~ns}$ is split to serve two analog-to-digital converters (ADCs) with a sampling rate sufficient to sample every pulse of the 216.7 $\mathrm{MHz}$ reference laser [10,38]. In both pairs the 16 bit ADCs sample the same signal with a phase shift to measure the relative height by subtraction of the base from peak $[10,38]$. By filtering of the samples it is ensured to find the relevant modulated pulse and specific unmodulated for normalization [10]. The raw arrival-time value is calculated in the field-programmable gate array (FPGA) on firmware level and transmitted to the low level rf system for the fast beam based feedback, which is time critical compared to the further processing in the DAQ computer [10].

\section{RF SIGNAL}

The limitations of current pickup structures are evident in the theoretical consideration of the time domain voltage signal. The image charge on the pickup surface is calculated by

$$
Q_{\mathrm{im}}(t)=\int_{-\infty}^{\infty} \frac{\lambda\left(z-c_{0} t\right) w(z)}{2 \pi r_{\mathrm{p}}} \mathrm{d} z
$$

with the line charge density $\lambda(t)$ of the ultrarelativistic bunch $\left(v \approx c_{0}\right)$, pickup width $w(z)$ and the distance between pickup surface and bunch $r_{\mathrm{p}}$ [39]. Because line charge density and pickup width are real quantities, by substitution of $z$ into a moving frame, Eq. (1) can be transformed into the cross-correlation

$$
Q_{\mathrm{im}}(t)=\frac{1}{2 \pi r_{\mathrm{p}}}(\lambda \star w)(t),
$$

which is readily solved numerically. This is true for any bunch and pickup form. In the special case of an even charge density, Eq. (1) is the convolution

$$
Q_{\mathrm{im}}(t)=\frac{1}{2 \pi r_{\mathrm{p}}}\left(\lambda^{\mathrm{even}} \circledast w\right)(t) .
$$

Equations (2) and (3) can be utilized in the numerical treatment and also for analytical solutions, which are accessible for many different cases. Some essential examples are covered in this section followed by the numerical approach.

\section{A. Analytical model}

Idealized representations of bunch and pickup surface allow to find an analytical solution for Eq. (1). A suitable representation that leads to a substantial simplification is given by a Gaussian bunch and a rectangular pickup surface. The stationary charge density of the Gaussian beam centralized on the $z$ axis then is

$$
\rho(x, y, z)=\delta(x) \delta(y) \lambda(z) .
$$

The line charge density is found by integration of the transverse area. A Gaussian bunch is given by

$$
\lambda(z)=\lambda_{0} \exp \left(-\frac{z^{2}}{2 \sigma_{\mathrm{z}}^{2}}\right)=Q_{\mathrm{b}} G\left(z, \sigma_{\mathrm{z}}\right),
$$

where $\lambda_{0}=Q_{\mathrm{b}} / \sqrt{2 \pi \sigma_{\mathrm{z}}^{2}}$ is a constant for normalization by the bunch charge, $Q_{\mathrm{b}}$ the total charge of one bunch, $\sigma_{\mathrm{z}}$ the rms length and $G\left(\tau, \sigma_{\tau}\right)$ the normalized Gaussian distribution function,

$$
G\left(\tau, \sigma_{\tau}\right)=\frac{1}{\sqrt{2 \pi} \sigma_{\tau}} \exp \left(-\frac{\tau^{2}}{2 \sigma_{\tau}^{2}}\right) .
$$

In the scope of this work, we denote

$$
\lambda(t):=\left.\lambda(z)\right|_{z=c_{0} t}=\frac{Q_{\mathrm{b}}}{c_{0}} G\left(t, \sigma_{\mathrm{t}}\right),
$$

using Eq. (5a), where the speed of light is incorporated into the rms length in units of time:

$$
\sigma_{\mathrm{t}}=\frac{\sigma_{\mathrm{z}}}{c_{0}}
$$

The rectangular pickup surface width is written as

$$
w(z)=w_{0} \Pi_{\ell}(z),
$$

where $\ell$ is the longitudinal extension of the pickup and

$$
\Pi_{\ell}(z)= \begin{cases}1 & |z| \leq \frac{1}{2} \ell \\ 0 & |z|>\frac{1}{2} \ell\end{cases}
$$

the rectangular function.

\section{Long bunch approximation}

In many applications, specifically with hadrons, it is reasonable to assume a bunch much longer than the pickup. This is treated in the long bunch approximation (LBA). The pickup form is insignificant in the LBA, hence a more general approach is used. For this purpose the parametrization of a finite pickup profile is

$$
w(z)=\tilde{w}(z) \chi_{[a, b]}(z),
$$

where $\chi_{[a, b]}(z)$ is the indicator function of the interval $[a, b]$, which bounds the pickup in the longitudinal direction, and 
$\tilde{w}(z)$ defines the pickup width at location $z$. The rectangular pickup in Eq. (7) is a special case of Eq. (9). The longitudinal characteristic dimension of the pickup is defined by $\ell=|a-b|$ and its surface area $A_{\mathrm{p}}$ by the integral

$$
A_{\mathrm{p}}=\int_{-\infty}^{\infty} \tilde{w}(z) \chi_{[a, b]}(z) \mathrm{d} z=\int_{a}^{b} \tilde{w}(z) \mathrm{d} z
$$

The image charge found by introducing Eq. (9) in Eq. (1) is

$$
Q_{\mathrm{im}}(t)=\frac{1}{2 \pi r_{\mathrm{p}}} \int_{-\infty}^{\infty} \lambda\left(z-c_{0} t\right) \tilde{w}(z) \chi_{[a, b]}(z) \mathrm{d} z
$$

which becomes

$$
Q_{\mathrm{im}}(t)=\frac{\lambda_{\mathrm{c}}}{2 \pi r_{\mathrm{p}}} \int_{a-c_{0} t}^{b-c_{0} t} \tilde{\lambda}(\zeta) \tilde{w}\left(\zeta+c_{0} t\right) \mathrm{d} \zeta
$$

by substitution of $\zeta=z-c_{0} t$ and factorizing $\lambda(\zeta)$ as the product of the constant term $\lambda_{\mathrm{c}}$, which has the units of a line charge density, and a dimensionless longitudinal distribution function $\tilde{\lambda}(\zeta)$. Despite the explicit dependence of the bunch form, the following derivation of the long bunch approximation only requires that the distribution $\tilde{\lambda}(z)$ can be written as

$$
\mathrm{T} \tilde{\lambda}\left(z ; c_{0} t\right)=\tilde{\lambda}\left(c_{0} t\right)+\tilde{\lambda}^{\prime}\left(c_{0} t\right)\left(z-c_{0} t\right)+\cdots,
$$

for all $z \in \mathbb{R}$ apart from an at most countable set of points. Here T $\tilde{\lambda}\left(z ; c_{0} t\right)$ is the Taylor expansion of $\tilde{\lambda}(z)$ at $c_{0} t$. The image charge can be written as an expansion as well,

$$
Q_{\mathrm{im}}(t)=\frac{\lambda_{\mathrm{c}}}{2 \pi r_{\mathrm{p}}} \sum_{n=0}^{\infty} q_{n}\left(c_{0} t\right),
$$

with

$q_{n}\left(c_{0} t\right)=\tilde{\lambda}^{(n)}\left(c_{0} t\right) \int_{a-c_{0} t}^{b-c_{0} t} \tilde{w}\left(\zeta+c_{0} t\right)\left(\zeta-c_{0} t\right)^{n} \mathrm{~d} \zeta$

For a long bunch, or equivalently a short pickup, it is justified to consider only the leading term, with $n=0$, if the reminder term is negligible, which implies the function to be constant in the rolling interval of length $\ell$. This is applicable if the following limit is true:

$$
\left|R_{0}\right|=\left|\tilde{\lambda}(z)-\tilde{\lambda}\left(c_{0} t\right)\right| \rightarrow 0 \quad \forall z \in\left[c_{0} t-a, c_{0} t+b\right]
$$

and justified for any given function at some point for $|a-b| \rightarrow 0$. Therefore, it is useful to introduce a scaling quantity $\mu$ for the bunch-pickup system, which controls the approximation for any Lipschitz continuous function. For this purpose because the derivative is bounded by $M_{1}=\sup \left\{\left|\tilde{\lambda}^{\prime}(t)\right|: t \in \mathbb{R}\right\}$, that is the Lipschitz constant of the distribution $\tilde{\lambda}(t)$, the following inequality gives an upper limit for the reminder $R_{0}$ :

$$
\tilde{\lambda}(z)-\tilde{\lambda}\left(c_{0} t\right) \leq M_{1}\left|z-c_{0} t\right| \leq M_{1}|b-a| .
$$

Therefore, the scaling is defined as

$$
\mu=M_{1}|b-a|=M_{1} \ell
$$

and the requirement in Eq. (14) is fulfilled for

$$
\mu \ll 1
$$

Applied on a Gaussian bunch according to Eq. (5a) it is

$$
\mu=\frac{1}{\sqrt{e}} \frac{l}{\sigma_{\mathrm{z}}} \ll 1
$$

Under this assumption, the image charge is $q_{0}$ and thus

$$
Q_{\mathrm{im}}^{\mathrm{LBA}}(t)=\frac{\lambda(t)}{2 \pi r_{\mathrm{p}}} \int_{a}^{b} \tilde{w}(z) \mathrm{d} z,
$$

which by Eq. (10) is

$$
Q_{\mathrm{im}}^{\mathrm{LBA}}(t)=\frac{A_{\mathrm{p}}}{2 \pi r_{\mathrm{p}}} \lambda(t) .
$$

This approximation is widespread and for example found in $[39,40]$. Equation $(20)$ is valid for any pickup which is short enough to neglect the change of line charge density along the pickup's length, which is ensured by requirement (14), independent of pickup and bunch form. The validity of this situation, i.e., of the long bunch approximation, is qualitatively controlled by condition (17). If this condition is satisfied, it is ensured that the requirement (14) is also met. Nonetheless Eq. (17) is a stricter condition and may be untrue for some functions that still are sufficiently described by the LBA. These requirements are met in many facilities, thus the theory is well developed and calculations for the image current and output voltage discussed in various publications on beam instrumentation and in particular beam position monitors as [40].

\section{Short bunch approximation}

In FEL applications as discussed in this work, the requirement of the long bunch approximation $\mu \ll 1$ is not fulfilled. Instead the bunch length is typically below 200 fs down to single digit fs and a standard pickup is in the range of some $\mathrm{mm}$, corresponding to $\geq 3.3 \mathrm{ps}$. With the bunch more then one sometimes up to 3 orders of 
magnitude shorter it is worthwhile to investigate the limiting case of ultrashort bunches, i.e.,

$$
\mu \gg 1 \Leftrightarrow \sigma_{\mathrm{z}} \ll \ell .
$$

Under this assumption in the short bunch approximation (SBA), because the normalized Gaussian is a Dirac sequence, Eq. (5a) is described by a delta distribution with the total charge $Q_{\mathrm{b}}$ concentrated at one point in space:

$$
\lim _{\sigma_{\mathrm{t}} \rightarrow 0} \lambda\left(z-c_{0} t\right)=Q_{\mathrm{b}} \delta\left(z-c_{0} t\right)
$$

Since the delta distribution is even, Eq. (1) is treated as a convolution, leading straightforward to

$$
Q_{\mathrm{im}}^{\mathrm{SBA}}(t)=\frac{Q_{\mathrm{b}} w_{0}}{2 \pi r_{\mathrm{p}}} \Pi_{2 t_{0}}(t),
$$

where

$$
t_{0}=\frac{\ell}{2 c_{0}}
$$

is the time it takes for the bunch center to pass half the pickup. The image current found by differentiation of $Q_{\mathrm{im}}^{\mathrm{SBA}}(t)$ is given by the following distribution:

$$
I_{\mathrm{im}}^{\mathrm{SBA}}(t)=\frac{Q_{\mathrm{b}} w_{0}}{2 \pi r_{\mathrm{p}}}\left[\delta\left(t+t_{0}\right)-\delta\left(t-t_{0}\right)\right] .
$$

The output voltage is simply derived for any given transfer function $H(\omega)$ with corresponding impulse response function $h(t)$ by the convolution with Eq. (25):

$$
U_{\mathrm{h}}^{\mathrm{SBA}}(t)=\left(h \circledast I_{\mathrm{im}}\right)(t) .
$$

Therefore, the voltage signal in time domain is

$$
U_{\mathrm{h}}^{\mathrm{SBA}}(t)=\frac{Q_{\mathrm{b}} w_{0}}{2 \pi r_{\mathrm{p}}}\left[h\left(t+t_{0}\right)-h\left(t-t_{0}\right)\right] .
$$

The preceding derivation of the short bunch limit is possible for all line charge density distributions in $L^{1}$, thus with finite total bunch charge $Q_{\mathrm{b}}$. The resulting voltage shape $U_{\mathrm{h}}^{\mathrm{SBA}}(t)$ is certainly independent of the infinitesimal short bunch form, but defined by the subtraction of the scaled impulse response function $h(t)$ shifted parallel in different directions.

In the following two idealized transfer functions are used to illustrate the implications of the ultrashort bunch. The two applied low-pass filters are a rectangular filter defined by

$$
H(\omega)=R \Pi_{2 \Omega_{\mathrm{c}}}(\omega)
$$

and a Gaussian filter defined by

$$
H(\omega)=R \exp \left(-\frac{\ln (2) \omega^{2}}{2 \Omega_{\mathrm{c}}^{2}}\right) .
$$

The cutoff frequency is $\Omega_{\mathrm{c}}$ and the filters are terminated with $\mathrm{R}$, usually $50 \Omega$. The corresponding time domain response functions are

$$
h_{\Pi}(t)=\frac{R \Omega_{\mathrm{c}}}{\pi} \operatorname{si}\left(\Omega_{\mathrm{c}} t\right)
$$

and

$$
h_{\mathrm{G}}(t)=\frac{R \tilde{\Omega}_{\mathrm{c}}}{\sqrt{2 \pi}} \exp \left(-\frac{\tilde{\Omega}_{\mathrm{c}}^{2}}{2} t^{2}\right),
$$

where $\tilde{\Omega}_{\mathrm{c}}=\Omega_{\mathrm{c}} / \sqrt{\ln (2)}$ is the cutoff frequency defined by a $1 / e$ decrease. The voltage signals $U_{\Pi}^{\mathrm{SBA}}(t)$ respectively $U_{\mathrm{G}}^{\mathrm{SBA}}(t)$ are found by substitution of the response function in Eq. (27). The resulting curves are shown in Fig. 2 assuming dimensions in the range of existing bunch arrival-time monitors. The characteristic curve, resembling the derivative of a Gaussian, is found at the center of both examples. An ideal rectangular filter induces an infinite ripple, which in some cases also reflects on the steep signal slope at the center, while the Gaussian is smooth.

The signal slope at the zero crossing

$$
S_{h, \mathrm{ZC}}=\dot{U}_{\mathrm{h}}\left(t_{\mathrm{ZC}}\right)
$$

is the figure of merit in BAM applications. The significant $\mathrm{ZC}$ is located at $t=0$ for both example filters. The respective slopes are

$$
S_{\Pi, \mathrm{ZC}}^{\mathrm{SBA}}=-\frac{R w_{0}}{\pi^{2} r_{\mathrm{p}}} Q_{\mathrm{b}} \frac{\Omega_{\mathrm{c}}}{t_{0}}\left[\cos \left(\Omega_{\mathrm{c}} t_{0}\right)-\operatorname{si}\left(\Omega_{\mathrm{c}} t_{0}\right)\right]
$$

and

$$
S_{\mathrm{G}, \mathrm{ZC}}^{\mathrm{SBA}}=-\frac{R A_{\mathrm{p}}}{2 \pi r_{\mathrm{p}}} \frac{Q_{\mathrm{b}}}{\sqrt{2 \pi}} \frac{\tilde{\Omega}_{\mathrm{c}}^{3}}{c_{0}}\left[\exp \left(-\frac{1}{2} \tilde{\Omega}_{\mathrm{c}}^{2} t_{0}^{2}\right)\right] .
$$

For the rectangular filter the slope is depending on the ratio of cutoff frequency and pickup length, with a periodical behavior caused by the bracket term. This term has local extrema of approximately \pm 1 . For simplicity it is sufficient to assume exactly \pm 1 , found at $\Omega_{\mathrm{c}} t_{0}=n \pi$, with $n \in \mathbb{N}$, although the real maximum is located somewhat before that.

For the Gaussian filter the slope is also depending on the ratio of cutoff frequency and pickup length. For an infinite as well as infinitesimal pickup the slope is vanishing. If the pickup length is varied at a fixed cutoff, the maximum slope is reached at $\tilde{\Omega}_{\mathrm{c}}=2 c_{0} / \ell$. If the length is kept constant and the cutoff frequency is varied, a factor of $\sqrt{3}$ has to be added on the equation's right hand side. For a lower cutoff frequency the signal width is increased, leading to 

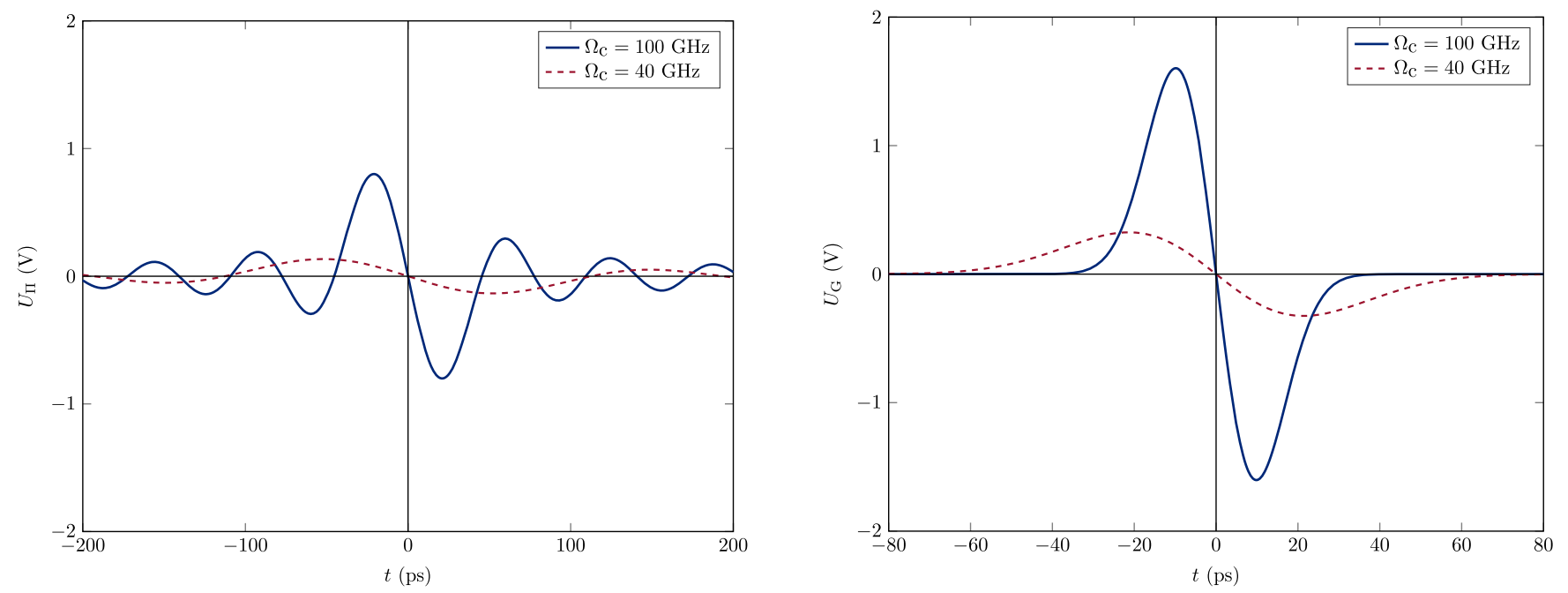

FIG. 2. Voltage signal in the SBA for two idealized filters. A rectangular (left) and a Gaussian filter (right) have been utilized, each at $100 \mathrm{GHz}$ (blue) and $40 \mathrm{GHz}$ (red) cutoff frequency. Bunch and geometry parameters are in a reasonable range for XFEL applications, e.g., $\sigma_{\mathrm{t}}=200 \mathrm{fs}, Q_{\mathrm{b}}=20 \mathrm{pC}, \ell=w_{0}=4.8 \mathrm{~mm}, r_{\mathrm{p}}=20 \mathrm{~mm}$ and $R=50 \Omega$.

distortion and partial annihilation of the signal. If the bandwidth is increased further, two distinctive peaks become visible leading to a lower gradient at zero crossing. The optimum for fixed cutoff frequency is

$$
S_{\mathrm{G}, \max }^{\mathrm{SBA}}=-\frac{1}{\sqrt{e}} \frac{R A_{\mathrm{p}}}{2 \pi r_{\mathrm{p}}} \frac{Q_{\mathrm{b}}}{\sqrt{2 \pi}} \frac{1}{c_{0}} \tilde{\Omega}_{\mathrm{c}}^{3} .
$$

This equals the maximum slope received by the short pickup and high cutoff frequency approximation, apart from the factor $1 / \sqrt{e}$ and the replacement of the reciprocal bunch width by the cutoff frequency, which are the corresponding decisive widths.

The real transfer function is neither rectangular nor Gaussian. In literature the pickup is usually modeled by an equivalent circuit of a resistor $\mathrm{R}$ parallel to the capacity $\mathrm{C}$ (RC circuit), with the image current $I_{\text {im }}$ serving as a current source $[40,41]$. The expected low-pass filter then gives

$$
H(\omega)=\frac{R}{1+i \omega R C},
$$

with cutoff frequency $\Omega_{\mathrm{RC}}=1 /(R C)$, as transfer function from current source $I_{\mathrm{im}}$ to the output voltage $U_{\mathrm{RC}}$. In time domain the impulse response function is

$$
h(t)=\frac{1}{C} \exp \left(-\frac{t}{R C}\right) \Theta(t),
$$

where $\Theta(t)$ denotes the Heaviside step function.

In order to calculate the output voltage with the equivalent circuit's transfer function the convolution with the image current in short bunch approximation according to Eq. (27) gives

$$
\begin{aligned}
U_{\mathrm{RC}}^{\mathrm{SBA}}(t)= & \frac{1}{C} \frac{Q_{\mathrm{b}} w_{0}}{2 \pi r_{\mathrm{p}}} \exp \left(-\frac{t+t_{0}}{R C}\right) \\
& \times\left[\Theta\left(t+t_{0}\right)-\exp \left(+\frac{2 t_{0}}{R C}\right) \Theta\left(t-t_{0}\right)\right],
\end{aligned}
$$

which corresponds to the capacitor experiencing the complete electric field of the infinitesimal bunch at once, leading to a compensating current, which is equivalent to the discharging of a capacitor. The decay curve is observed until the coasting beam reaches the end of the pickup. Then the effect of the bunch's electric field on the pickup suddenly ends and the initial voltage jump is reversed. Afterwards the charges start flowing back, leading to a continuation of the exponential voltage decay. Therefore slope at zero crossing, found at $t=t_{0}$, is an infinite decrease.

\section{General solution for the image current}

In case none of the preceding approximations is sufficient a general solution for Eq. (1) is needed. It is found straightforward for Gaussian bunches and rectangular pickups. Applying the shapes defined in Eqs. (5a) and (7) without any approximation leads to

$$
Q_{\mathrm{im}}(t)=\frac{w_{0} Q_{\mathrm{b}}}{2 \pi r_{\mathrm{p}}} \int_{-\ell / 2}^{\ell / 2} G\left(z-c_{0} t, \sigma_{\mathrm{z}}\right) \mathrm{d} z
$$

This integral is readily solved, giving

$Q_{\mathrm{im}}(t)=\frac{1}{2} \frac{w_{0}}{2 \pi r_{\mathrm{p}}} Q_{\mathrm{b}}\left[\operatorname{erf}\left(\frac{\frac{\ell}{2}-c_{0} t}{\sqrt{2} \sigma_{\mathrm{z}}}\right)+\operatorname{erf}\left(\frac{\frac{\ell}{2}+c_{0} t}{\sqrt{2} \sigma_{\mathrm{z}}}\right)\right]$ 

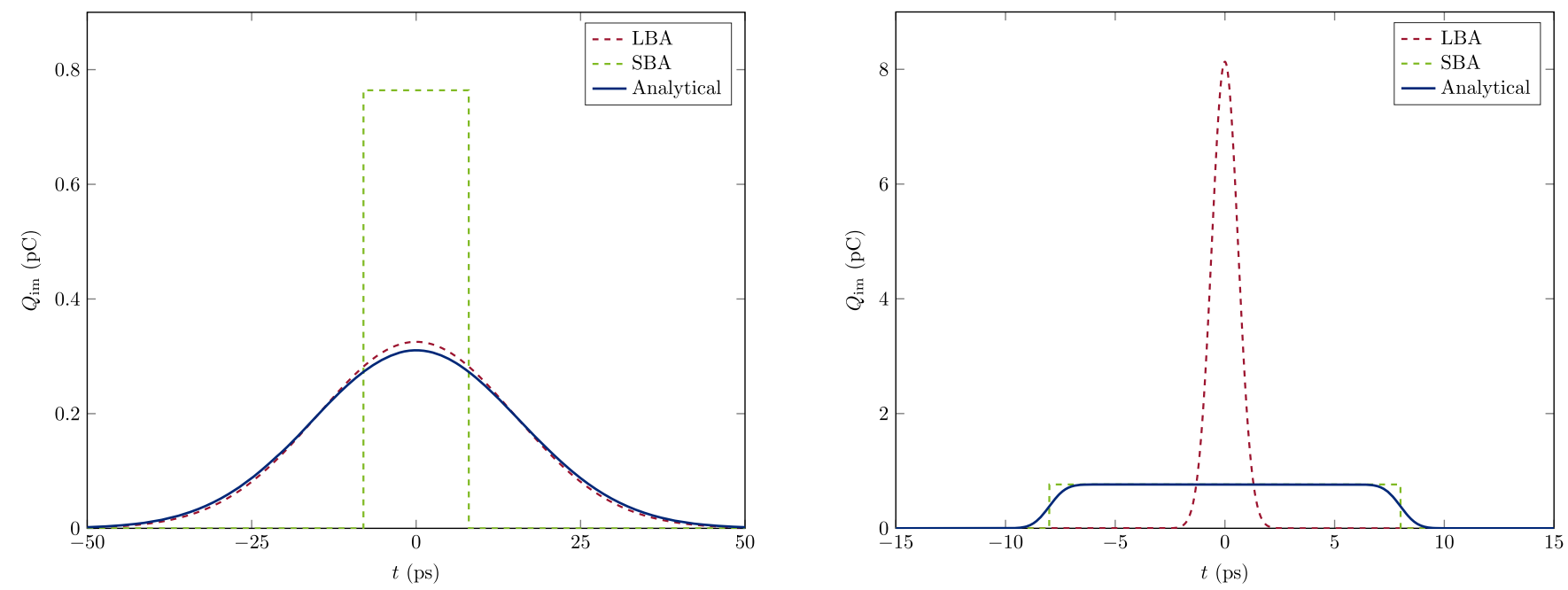

FIG. 3. Image charge by a Gaussian bunch on a rectangular pickup surface according to the LBA in Eq. (20) (dashed red), the SBA in Eq. (23) (dashed green) and the analytical solution in Eq. (37) (solid blue) for two different bunch lengths. One close to the LBA regime, with $\mu \approx 0.65$ (left) and one closer to the SBA regime, with $\mu \approx 16.2$ (right). Bunch and geometry parameters are in a reasonable range for applications of button pickups, e.g., $\sigma_{\mathrm{t}}=0.6 \mathrm{ps}$ respectively $\sigma_{\mathrm{t}}=15 \mathrm{ps}, Q_{\mathrm{b}}=20 \mathrm{pC}, \ell=w_{0}=4.8 \mathrm{~mm}$ and $r_{\mathrm{p}}=20 \mathrm{~mm}$. In the EuXFEL even shorter bunches occur, further reducing the discrepancy between SBA and analytical solution at the edges.

for the image charge induced on the pickup by the coasting beam.

Figure 3 shows exemplary results of the analytical description in Eq. (37) (blue) compared to the LBA, as in Eq. (20) (dashed red), and the SBA, as in Eq. (23) (dashed green), for two different values of the scaling factor $\mu$. In case of a short bunch, $\mu \approx 16.2$ (right), when the coasting bunch is almost completely enclosed longitudinally by the pickup surface, a flat section is notable in the analytical result, which is in accordance with the SBA, while the LBA gives a sharp peak. In case of long bunches the curves found analytical and by LBA converge, but the SBA in Eq. (23) still gives a flat center with steep edges. Therefore both approximations are well suited for their cases, but differ significantly apart from that.

Differentiation of Eq. (37) gives the general image current

$$
I_{\mathrm{im}}(t)=\frac{w_{0}}{2 \pi r_{\mathrm{p}}} Q_{b}\left[G\left(t+t_{0}, \sigma_{\mathrm{t}}\right)-G\left(t-t_{0}, \sigma_{\mathrm{t}}\right)\right]
$$

with $t_{0}$ defined in Eq. (24) and bunch width $\sigma_{\mathrm{t}}$ expressed in units of time according to (6). The first Gaussian represents the incoming charges introduced by the arriving bunch, the other is caused by the bunch leaving the effective area. This result can be understood as a generalization to the current found in the short bunch approximation. If the delta distributions are replaced by Gaussians, the factor differs by the normalization $1 / \sqrt{2 \pi \sigma_{\mathrm{t}}^{2}}$. Consistently the general solution is readily converted into Eq. (25) by the limit of an ultrashort bunch length.

The general voltage signal measured at resistor $\mathrm{R}$ is found by the known transfer function based on the pickups equivalent circuit, see Eq. (33). It is determined by the convolution of the general image current, Eq. (38), and the impulse response function, Eq. (34). The result, also mentioned in [41], is

$$
\begin{aligned}
U_{\mathrm{RC}}(t)= & \frac{1}{2} \frac{1}{C} \frac{w_{0}}{2 \pi r_{\mathrm{p}}} Q_{\mathrm{b}} \exp \left[\frac{1}{2}\left(\frac{\sigma_{\mathrm{t}}}{R C}\right)^{2}\right]\left\{\exp \left(-\frac{t+t_{0}}{R C}\right)\right. \\
& \times\left[1+\operatorname{erf}\left(\frac{t+t_{0}}{\sqrt{2} \sigma_{\mathrm{t}}}-\frac{\sigma_{\mathrm{t}}}{\sqrt{2} R C}\right)\right] \\
& \left.-\exp \left(-\frac{t-t_{0}}{R C}\right)\left[1+\operatorname{erf}\left(\frac{t-t_{0}}{\sqrt{2} \sigma_{\mathrm{t}}}-\frac{\sigma_{\mathrm{t}}}{\sqrt{2} R C}\right)\right]\right\} .
\end{aligned}
$$

The voltage curve shows a very different behavior for different parameter choice, which is not immediately accessible in Eq. (39). The two introduced limiting cases for $\mu \ll 1$ and $\mu \gg 1$ are in accordance with the general solution. For an intermediate value of $\mu$, it is possible to find an approximation of Eq. (39) by assuming extreme values for the cutoff frequency or in particular the capacity, as presented in the next section. The limiting cases and the transitional behavior are exemplified in Fig. 4. On the left side bunch and pickup length approach the LBA, $\mu \approx 0.7$. In contrast the right image contains signals for a relatively short bunch with $\mu \approx 24$, looking like the SBA. In both plots the capacitance is varied starting at $0.03 \mathrm{pF}$, close to the so-called inductive case (red) to $1 \mathrm{pF}$ respectively $4 \mathrm{pF}$, which is around the capacitive case (blue). The corresponding cutoff frequencies are $\Omega_{\mathrm{RC}}=0.67 \mathrm{THz}$ and $5 \mathrm{GHz}$ respectively $20 \mathrm{GHz}$. The transition between both cases is indicated with stripped lines. 

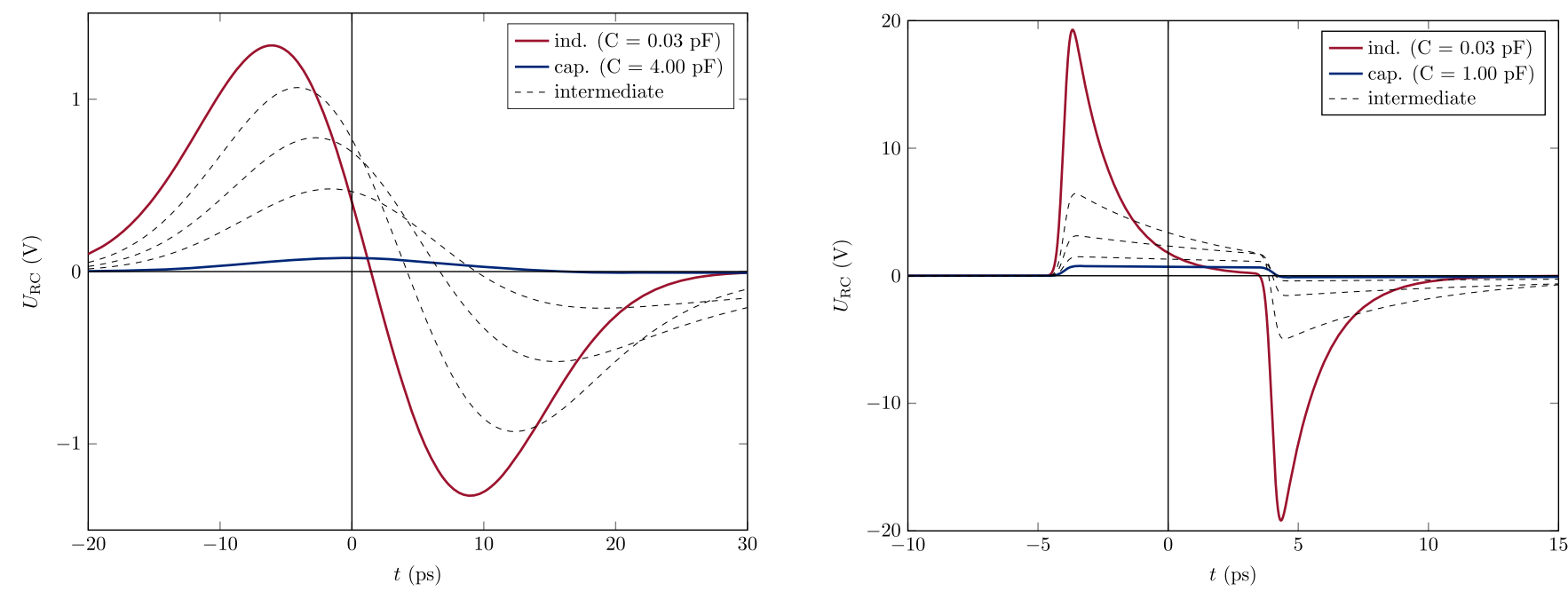

FIG. 4. Voltage signal according to Eq. (39) with dimensions in the range of the LBA with $\mu \approx 0.7$ (left) and SBA with $\mu \approx 24$ (right). The capacity is varied from almost the inductive limit (red) up to the capacitive case (blue) with three intermediate steps (dashed). Bunch and geometry parameters are in a reasonable range for XFEL applications, e.g., $\sigma_{\mathrm{t}}=7 \mathrm{ps}$ (left) respectively $\sigma_{\mathrm{t}}=0.2 \mathrm{ps}($ right), $Q_{\mathrm{b}}=20 \mathrm{pC}, \ell=2.4 \mathrm{~mm}, w_{0}=4.8 \mathrm{~mm}$ and $r_{\mathrm{p}}=20 \mathrm{~mm}$. In other applications, when the capacitive case is required, the limiting case can be achieved by increasing the terminating resistor $\mathrm{R}$, to counter the $1 / C$ dependence in Eq. (39), see Fig. 3 (a) in [41].

The BAM resolution depends on the signal slope at the zero crossing (ZC). The time dependent signal slope found by derivation of Eq. (39)

$$
\dot{U}_{\mathrm{RC}}(t)=\Omega_{\mathrm{RC}}\left[R I_{\mathrm{im}}(t)-U_{\mathrm{RC}}(t)\right]
$$

At the time of zero crossing $t_{\mathrm{ZC}}$ the voltage $U_{\mathrm{RC}}\left(t_{\mathrm{ZC}}\right)$ is zero by definition. Therefore, the signal slope at the zero crossing is only defined by the image current

$$
S_{\mathrm{RC}, \mathrm{ZC}}=\dot{U}_{\mathrm{RC}}\left(t_{\mathrm{ZC}}\right)=\frac{1}{C} I_{\mathrm{im}}\left(t_{\mathrm{ZC}}\right) .
$$

Because $I_{\mathrm{im}}(t=0)$ is zero, a zero crossing at $t_{\mathrm{ZC}}=0$ would entail a gradient of zero, but the zero crossing is located at $t_{\mathrm{ZC}} \neq 0$ for any $C>0$.

\section{Voltage by frequency regions}

In many theoretical approaches the voltage signal is analyzed in two special cases defined by frequency regions. In the so-called capacitive limit, the capacity $\mathrm{C}$ is large, respectively the cutoff frequency approaches zero $\Omega_{\mathrm{RC}} \rightarrow 0$, hence all exponential functions approach one and the first term of each error function is predominant. This leads to a great simplification of the output voltage, which is

$$
U_{\mathrm{RC}}^{\mathrm{cap}}(t)=\frac{1}{C} Q_{\mathrm{im}}(t)
$$

Therefore, the signal in the limit of a infinitesimal cutoff frequency resembles a charge source [41]. The limit for an infinitesimal capacity, the inductive case [41] with
$\Omega_{R C} \rightarrow \infty$, is straightforward for the frequency domain transfer function Eq. (33), which becomes the constant R, and thus $h(t)=R \delta(t)$ in time domain. The voltage is then

$$
U_{\mathrm{RC}}^{\mathrm{ind}}(t)=R I_{\mathrm{im}}(t),
$$

resembling a current source [41]. In this case, the zero crossing is exactly at $t=0$ and for a finite pickup length the signal slope is nonzero in a seeming contradiction to Eq. (41), but the denominator $\mathrm{C}$ is zero as well.

\section{Maximum voltage and signal slope}

For deployment in a BAM, maximum voltage and signal slope are the key features of the pickup. Moreover, a thorough understanding of the decisive parameters is crucial. The general formulation in Eq. (39) confirms the proportionality to the bunch charge and pickup width as well as reciprocal to the distance between bunch and pickup

$$
U_{\mathrm{RC}, \max } \propto Q_{\mathrm{b}} \frac{w_{0}}{r_{\mathrm{p}}}
$$

and

$$
S_{\mathrm{RC}, \max } \propto Q_{\mathrm{b}} \frac{w_{0}}{r_{\mathrm{p}}} .
$$

The proportionality to the bunch charge was experimentally proven for the signal slope of the cone-shaped pickups at FLASH [34]. The inverse proportionality to $r_{\mathrm{p}}$ has to be analyzed primarily in simulations, since these measurements are expensive and limited by the facilities design parameters. Nevertheless, the measurements reported 
in $[20,21]$, which were done at the SITF and SwissFEL with different beam line diameters, support this result.

Another dependency observed in simulations is on the ratio of pickup and bunch lengths. The maximum voltage approaches its maximum asymptotically and deviates only slightly from the value for a pickup longer than a few bunch lengths $[24,41]$. This also effects the signal slope, as the maximum voltage barely changes in this region while both extrema drift apart, leading to a decreasing slope [24]. In Eq. (39) it is readily shown that the voltage vanishes for $t_{0}=0$, which is identical to $\ell=0$. To describe the behavior for any other ratio, the approximations must be utilized. In case of the pure charge source the maximum voltage found at $t_{\max }=0$ is

$$
\max \left(U_{\mathrm{RC}}^{\mathrm{cap}}\right)=\frac{1}{C} \frac{w_{0} Q_{\mathrm{b}}}{2 \pi r_{\mathrm{p}}} \operatorname{erf}\left(\frac{\ell}{\sqrt{8} \sigma_{\mathrm{z}}}\right)
$$

[41]. For a pure current source $t_{\max }$ must adhere to

$$
t_{0}=t_{\max } \tanh \left(\frac{t_{0}}{\sigma_{\mathrm{t}}} \frac{t_{\max }}{\sigma_{\mathrm{t}}}\right)
$$

which is found by setting $\dot{U}_{\mathrm{RC}}^{\text {ind }}=0$. Two useful approximations give the positions of extrema for a large or negligible argument of the hyperbolic tangent, which are $t_{\max } \approx \pm \sigma_{\mathrm{t}}$ for the LBA with $t_{0} \ll \sigma_{\mathrm{t}}$ respectively $t_{\max } \approx$ $\pm t_{0}$ for the SBA with $t_{0} \gg \sigma_{\mathrm{t}}$. The corresponding maximum voltages are for a short pickup

$$
\max \left(U_{\mathrm{RC}, t_{0} \ll \sigma_{\mathrm{t}}}^{\mathrm{LBA}, \sigma_{1}}\right) \approx \frac{R}{\sqrt{e}} \frac{A_{\mathrm{p}}}{2 \pi r_{\mathrm{p}}} \frac{Q_{\mathrm{b}}}{\sqrt{2 \pi} c_{0} \sigma_{\mathrm{t}}^{2}}=\mu \hat{U}_{0}^{\mathrm{ind}},
$$

with

$$
\hat{U}_{0}^{\text {ind }}=R \frac{w_{0}}{2 \pi r_{\mathrm{p}}} \frac{Q_{\mathrm{b}}}{\sqrt{2 \pi} \sigma_{\mathrm{t}}},
$$

and for a long pickup

$$
\max \left(U_{\mathrm{RC}, t_{0} \gg \sigma_{\mathrm{t}}}^{\mathrm{SBA}, \sigma_{1}}\right) \approx \hat{U}_{0}^{\text {ind }}\left[1-\exp \left(-\frac{l^{2}}{2 \sigma_{\mathrm{z}}^{2}}\right)\right]
$$

The first term is the maximum voltage for $\ell \rightarrow \infty$, which is justified for $\ell>3 \sigma_{\mathrm{z}}$ with the Gaussian bunch. In the cases described by Eqs. (46) and (50) the maximum voltage saturates for a pickup length of a few $\sigma_{\mathrm{z}}$, whereas in the realms of LBA, with an infinitesimal pickup, the maximum voltage goes linear with its length.

Regarding the BAM resolution a high signal slope is desired. The main proportionality is already given by Eq. (45), but in the inductive limit a general solution is possible. The slope at the zero crossing is

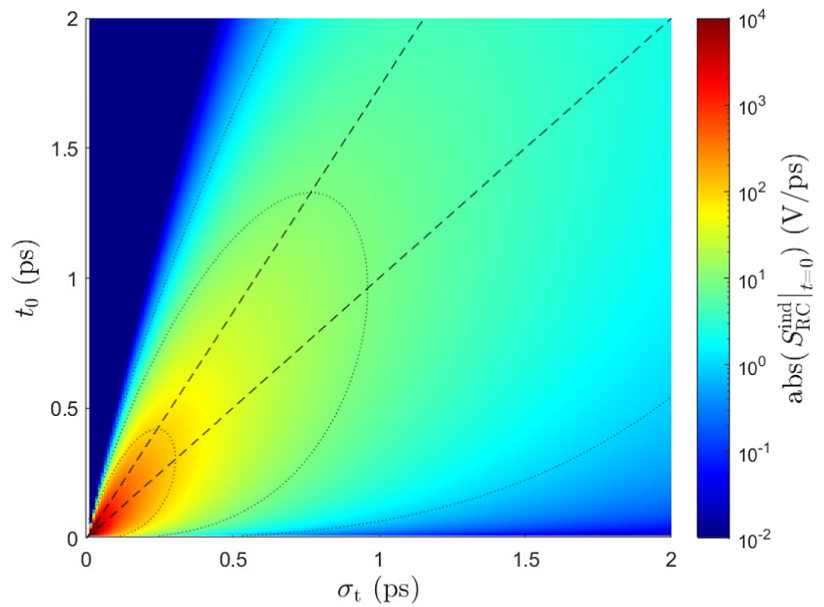

FIG. 5. Absolute signal slope at zero crossing according to Eq. (51) as a function of $t_{0}$ and $\sigma_{\mathrm{t}}$ in the interval from 0 to $2 \mathrm{ps}$. The dotted contours mark 1,10 and $100 \mathrm{~V} \mathrm{ps}^{-1}$ and dashed lines indicate $t_{0}=\sigma_{\mathrm{t}}$ and $t_{0}=\sqrt{3} \sigma_{\mathrm{t}}$.

$$
\left.S_{\mathrm{RC}}^{\mathrm{ind}}\right|_{t=0}=-R Q_{\mathrm{b}} \frac{w_{0}}{\pi r_{\mathrm{p}}} \frac{t_{0}}{\sigma_{\mathrm{t}}^{2}} G\left(t_{0}, \sigma_{\mathrm{t}}\right) .
$$

This result is visualized in Fig. 5. For a fixed bunch length, the optimum is reached at $t_{0}=\sigma_{\mathrm{t}}$, whereas for a fixed pickup length the best result is found at $t_{0}=\sqrt{3} \sigma_{\mathrm{t}}$. At $t_{0}=$ $\sigma_{\mathrm{t}}=0$ is a pole, therefore reduction of both dimensions is favorable in the case of a pure charge source, but in real applications the bunch length is in a fixed range specific for the facility and foreseen experiments.

\section{Bandwidth limitation}

The measured signal slope is limited by the bandwidth according to the uncertainty principle. The product of a signal's rise time $\tau$ as response to a step function and the frequency bandwidth $\Delta f$ satisfies $^{1}$

$$
\tau \Delta f=1 .
$$

This rise time serves as an upper limit and can be expressed by the maximum slope $S_{\max }$ and the peak-to-peak voltage $U_{\mathrm{PP}}$ with $\tau=U_{\mathrm{PP}} / S_{\max }$ giving

$$
S_{\max } \leq U_{\mathrm{pp}} \Delta f .
$$

This limitation affects the resolution of a transmitted signal. It determines the highest possible slope allowed in the response function, but if the slope of an incoming signal is well below this limit, a rise in bandwidth might even have a negative effect on the output signal slope, as already shown for the idealized Gaussian filter in the SBA.

\footnotetext{
${ }^{\mathrm{I}}$ Denote that the right-hand side of the related uncertainty inequalities by Küpfmüller [42] and Gabor [43] depend on the definitions of bandwidth and duration.
} 


\section{Circular pickup}

In all prior considerations the pickup was assumed rectangular, which is not in accordance with many realworld applications. From Fig. 4 and the formulation of the SBA it is reasonable to assume that the rectangular model is sufficient for long bunches, but its shape is significantly reflected in the voltage signal for shorter bunches. A general analytical solution for the circular surface of radius $r_{\mathrm{B}}$, defined by

$w(z)=2 \sqrt{r_{\mathrm{B}}^{2}-z^{2}} \Pi_{2 r_{\mathrm{B}}}= \begin{cases}2 \sqrt{r_{\mathrm{B}}^{2}-z^{2}} & |z| \leq r_{\mathrm{B}} \\ 0 & |z|>r_{\mathrm{B}}\end{cases}$

[39], is not available and numerical methods have to be utilized. Nonetheless, some special cases are accessible by analysis. One option is the SBA, with a bunch according to Eq. (22). In this case the image current is calculated by the convolution of bunch shape $\lambda(z)$ and distributional derivative of the pickup profile $w(z)$. It is expressed by

$$
\begin{aligned}
I_{\mathrm{im}}^{\mathrm{SBA}}(t)= & -\frac{c_{0}^{2} Q_{\mathrm{b}}}{\pi r_{\mathrm{p}}} \frac{t}{\sqrt{r_{\mathrm{B}}^{2}-\left(c_{0} t\right)^{2}}} \Pi_{2 r_{\mathrm{B}}} \\
& +\frac{c_{0} Q_{\mathrm{b}}}{\pi r_{\mathrm{p}}} \sqrt{r_{\mathrm{B}}^{2}-\left(c_{0} t\right)^{2}} \delta\left(c_{0} t+r_{\mathrm{B}}\right) \\
& -\frac{c_{0} Q_{\mathrm{b}}}{\pi r_{\mathrm{p}}} \sqrt{r_{\mathrm{B}}^{2}-\left(c_{0} t\right)^{2}} \delta\left(c_{0} t-r_{\mathrm{B}}\right) .
\end{aligned}
$$

For the intended application, only the center around $t=0$ is relevant, where the contribution of both dirac deltas is negligible. In the inductive limit, interesting for synchronization, the voltage is given by Eq. (43), with the zero crossing exactly at $t=0$ and a signal slope of

$$
\left.S_{\mathrm{RC}}^{\mathrm{SBA}, \text { ind }}\right|_{t=0}=-R \frac{c_{0}^{2} Q_{\mathrm{b}}}{\pi r_{\mathrm{p}}} \frac{1}{r_{\mathrm{B}}} .
$$

The slope is inversely proportional to the radius of the button pickup, hence a smaller button is favorable, though this approximation is only valid for a button much larger than the bunch.

\section{Summary}

An analytical solution for the voltage signal is readily found, which is valid for any bunch or pickup length and for any value of the lumped elements in the equivalent circuit representing the physical pickup. For different limiting cases, as the SBA, LBA, capacitive as well as inductive limit, appropriate expressions exist, which are easier to work with.

Nonetheless, some evaluations were carried out for the general solution as well. First of all, the highly relativistic bunch is assumed Gaussian and centered on the longitudinal axis. Usually the pickup was treated as a rectangular surface with the same curvature as the beampipe, to keep the radial distance to the beam constant. Fringing fields caused by the gap between pickup surface and the beampipe are neglected, but might be considered sufficiently by a constant factor depending on the geometry. The equivalent circuit was idealized as RC elements in parallel.

The most critical approximation is the rectangular pickup surface. The solution is still useful in the range of medium to long bunches, but the form becomes a significant factor for $\mu \gg 1$. There are numerical methods for arbitrary pickup surfaces.

\section{B. Numerical solution}

A numerical solution of Eq. (1) is accessible for any bunch or pickup shape, to study the signal in cases where the prior assumptions are not valid. While the Gaussian bunch in Eq. (5a) usually is a good approximation, the button pickup has a circular surface with a width given by Eq. (54). In Fig. 4 the pickup form is apparent in the signal shape in case of short bunches. An analytical solution was only determined in the SBA with an inductive pickup. It is therefore crucial to find a general description considering the pickup form, which is possible by numerical methods. Acknowledging that for any even charge distribution, Eq. (1) corresponds to a convolution, the image charge is readily calculated in frequency domain by the convolution theorem. It is

$$
Q_{\mathrm{im}}(\omega)=\mathrm{FFT}\left[\frac{\lambda\left(c_{0} t\right)}{2 \pi r_{\mathrm{p}}}\right] \operatorname{FFT}\left[w\left(c_{0} t\right)\right],
$$

where FFT is the fast Fourier transform. In case of an uneven charge density distribution it is necessary to exchange $\operatorname{FFT}\left[\lambda\left(c_{0} t\right)\right]$ by the complex conjugate $\overline{\operatorname{FFT}\left[\lambda\left(c_{0} t\right)\right]}$. By inverse FFT the time domain image charge is

$$
Q_{\mathrm{im}}(t)=\mathrm{FFT}^{-1}\left[Q_{\mathrm{im}}(\omega)\right]
$$

[39]. The image current is

$$
I_{\mathrm{im}}(t)=\mathrm{FFT}^{-1}\left[i \omega Q_{\mathrm{im}}(\omega)\right]
$$

and the voltage at the output of a system with transfer function $H(\omega)$

$$
U_{\mathrm{h}}(t)=\mathrm{FFT}^{-1}\left[H(\omega) I_{\mathrm{im}}(\omega)\right] .
$$

This method works well for finite inputs. Residual values, e.g., of the response function, at the end of the time interval lead to unphysical effects. Therefore, the time interval and sample frequency must be chosen with care.

The voltage signal calculated with a parameter set in the range of the operational parameters at the EuXFEL is pictured in Fig. 6. The dashed blue line represents the numerical result for a rectangular pickup, which is in good 

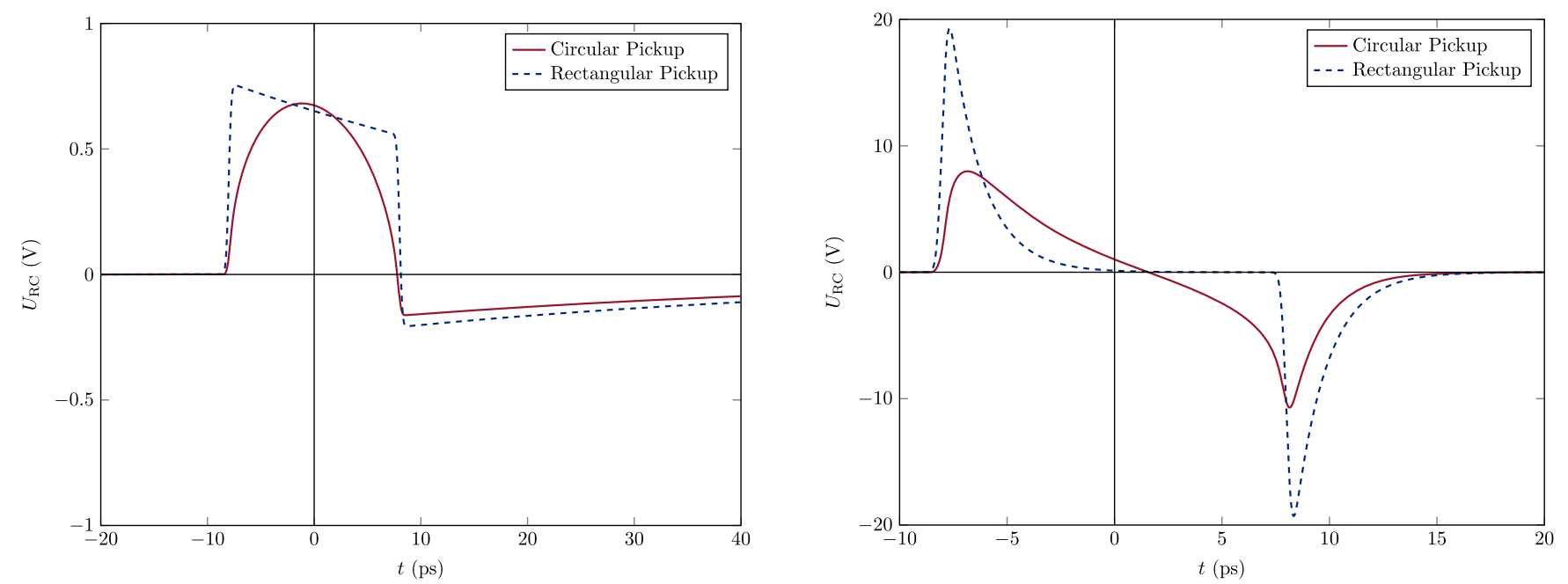

FIG. 6. Voltage signal of a rectangular (dashed blue) and a round (solid red) pickup surface calculated with MATLAB ${ }^{\circledR}$ according to Eqs. (57) to (60). Analog to the analytic approach, a Gaussian bunch and an RC filter were used. The dimensions are in range of the SBA with $\mu \approx 49$ and at the capacitive limit (left) respectively inductive limit (right). Bunch and geometry parameters, apart from the pickup length, are the same as in Fig. 4 (right), e.g., $R=50 \Omega, C=1 \mathrm{pF}$ (left) respectively $C=0.03 \mathrm{pF}\left(\right.$ right), $\sigma_{\mathrm{t}}=0.2 \mathrm{ps}, Q_{\mathrm{b}}=20 \mathrm{pC}$, $\ell=w_{0}=r_{\mathrm{B}}=4.8 \mathrm{~mm}$ and $r_{\mathrm{p}}=20 \mathrm{~mm}$.

agreement with the analytical version in Fig. 4 (right). The red line shows the signal by a circular pickup.

\section{LOW CHARGE MODE}

For new experiments in the EuXFEL, with a low charge mode $(\leq 1 \mathrm{pC})$, the second generation pickups are incapable of providing the required signal for fs resolution. Therefore, the BAM will be upgraded with a novel pickup structure and new EOM.

\section{A. Planned signal improvement}

New layouts are restrained in each facility by design regulations and previous design choices. For the next BAM upgrade in the EuXFEL a smaller pipe diameter is now permitted. The possible reduction from $40.5 \mathrm{~mm}$ [23] to $10 \mathrm{~mm}$ gives a potentially fourfold signal increase, by relation (45). When additionally the bandwidth is raised, according to inequality (53), one can expect a total improvement by 1 order of magnitude. Moreover, it is possible to combine multiple signals and to shorten the lossy rf path for further improvement.

The downside of these changes is a shorter dynamical range and a higher damage risk due to increased likeliness of direct beam impacts and high voltages at high charge mode. Special attention must be given to machine protection for all subsequent components.

\section{B. Aperture reduction}

A straightforward option for improvement, which may serve as a reference to quantify the potential benefits of new pickup designs, is the sole reduction of the beampipe aperture. This method is in agreement with the observations at the Paul Scherrer Institute. There the first generation pickup design was adapted for lower beampipe diameters at SwissFEL [20,21]. A diameter of $10 \mathrm{~mm}$, as now granted for the EuXFEL, is possible in combination with the smaller first-generation cone-shaped pickup and in between the 8 and $16 \mathrm{~mm}$ BAMs currently in use at the SwissFEL [21]. The second-generation pickups cannot be installed in a four-pickup configuration with this diameter due to their dimensions, as the cut-outs would overlap. A simulation with the wakefield solver of CST PARTICLE STUDIO ${ }^{\text {TM }}$ yields a slope of $1746 \mathrm{mV} \mathrm{ps}^{-1}$ with $13.7 \mathrm{~V}$ peak-to-peak voltage and a dynamical range of $12.4 \mathrm{ps}$ at the nominal $20 \mathrm{pC}$. The ringing of the signal pictured in Fig. 7 initially is significantly higher but decreases rapidly during the first $0.3 \mathrm{~ns}$. The increase seemingly exceeds the expectations for a fourfold radius reduction because the first generation pickups already exhibit a higher slope [23].

Despite additional influences, the comparison of the measured resolution at the SITF with $40.5 \mathrm{~mm} \mathrm{[20]} \mathrm{and} \mathrm{the}$ SwissFEL with $16 \mathrm{~mm}$ [21] diameter substantiates this notion and may be supported by an expected improvement for SwissFEL's $8 \mathrm{~mm}$ beam line [21]. So far no measurements with $8 \mathrm{~mm}$ have been published.

\section{90-GHz cone-shaped pickup}

We proposed a concept with pickups scaled to support up to $90 \mathrm{GHz}$ in 2019 with the dimensions specified in the first column of Table I [25]. This pickup was simulated afterwards in a BAM-like setup, with four identical pickups equally distributed around a pipe section, omitting the proposed signal combination. In addition, the former bunch parameters have been initially used. These are a 

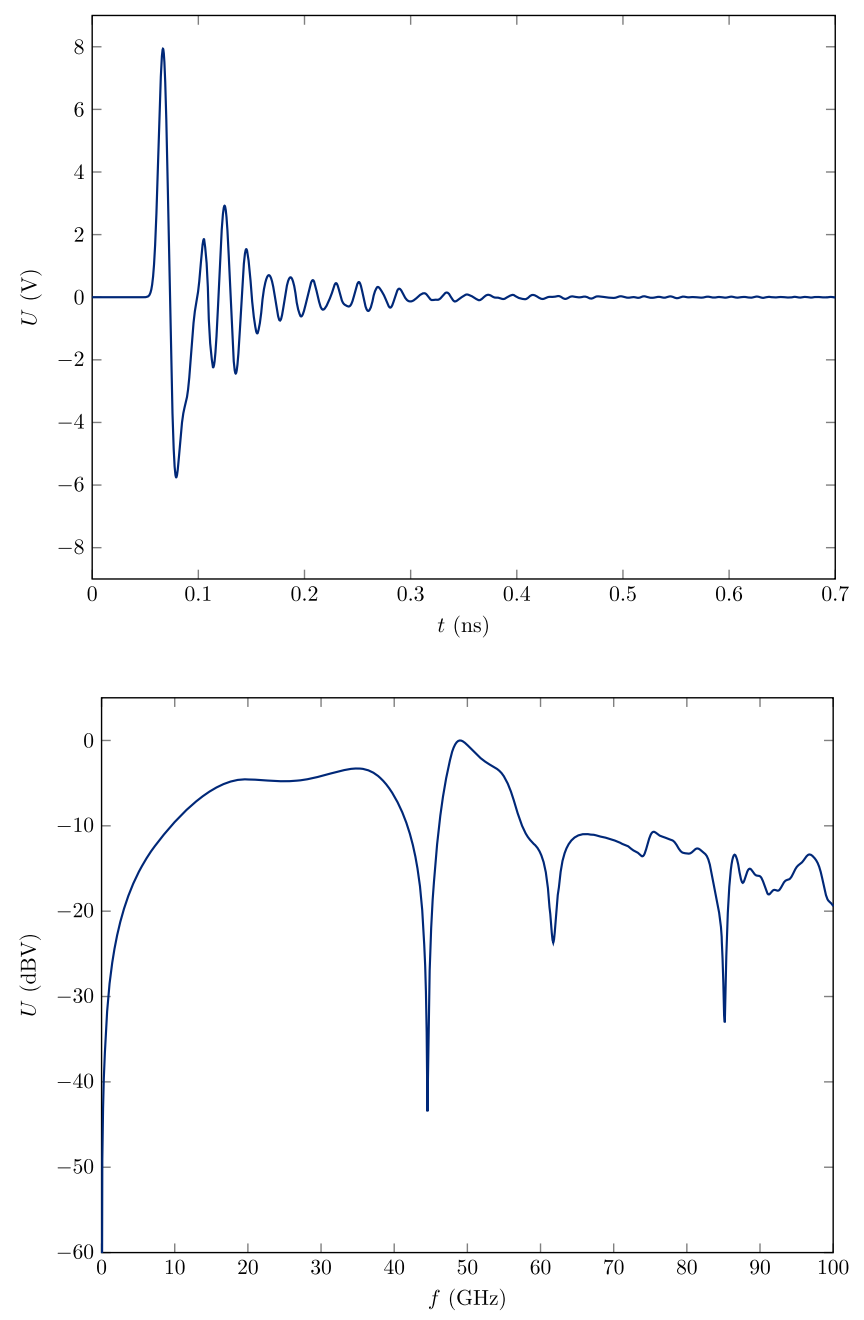

FIG. 7. Simulated signal in time domain (top) and its normalized spectrum (bottom) taken at the end of the vacuum feedthrough of a single pickup with $10 \mathrm{~mm}$ minimum distance, adapted from [44].

ultrarelativistic $\left(v=c_{0}\right)$ Gaussian bunch with $\sigma_{\mathrm{z}}=1 \mathrm{~mm}$ and a charge of $20 \mathrm{pC}$. Therefore, the voltage can be compared to the well-described state-of-the-art pickups according to Angelovski et al. [24,34].

The bipolar signal pictured in Fig. 8 has a peak-to-peak voltage of $3.52 \mathrm{~V}$. The peaks are separated by $8.01 \mathrm{ps}$, more than doubling the slope to $722.4 \mathrm{mV} \mathrm{ps}^{-1}$. Though the pipe radius was decreased nearly by a factor of 4 , the gain is only by 2.4. The increased bandwidth leads to a reduced rise time of 7.9 ps but cannot compensate for the missing protrusion and the smaller active area of base diameter $1.02 \mathrm{~mm}$. Thus, the pickup cannot outperform the first generation, but a smaller peak-to-peak voltage gives advantages in machine protection.

Reducing the bunch charge to $1 \mathrm{pC}$ accordingly gives a slope of $36.2 \mathrm{mV} \mathrm{ps}^{-1}$, which undershoots the minimum target by about a factor of 4 . For a further increase the combination of more than two signals is planned. By the
TABLE I. Specifications of the $90 \mathrm{GHz}$ pickup [25], the modified pickup (Second generation) [34] and the original (First generation) [23].

\begin{tabular}{|c|c|c|c|}
\hline & Draft'19 & $\begin{array}{c}\text { Second } \\
\text { generation }\end{array}$ & First generation \\
\hline Cutout diameter (mm) & 1.00 & 1.62 & 1.62 \\
\hline $\begin{array}{l}\text { Tapered cutout } \\
\text { diameter }(\mathrm{mm})\end{array}$ & 2.26 & 13.6 & 5.60 \\
\hline Cone diameter $(\mathrm{mm})$ & 0.45 & 0.70 & 0.70 \\
\hline $\begin{array}{l}\text { Tapered cone } \\
\text { diameter }(\mathrm{mm})\end{array}$ & 1.02 & 6 & 2.42 \\
\hline Cone height (mm) & $1^{\mathrm{a}}$ & 6 & 6 \\
\hline Protrusion (mm) & $1^{\mathrm{a}}$ & 1 & 0 \\
\hline Relative permittivity & 3.75 & 4.1 & 4.1 \\
\hline Line impedance $(\Omega)$ & 50.0 & 50.0 & 50.0 \\
\hline
\end{tabular}

${ }^{\mathrm{a}}$ Not specified in the publication.

combination of eight pickups, without any phase shift and $3 \mathrm{~dB}$ attenuation at each stage, a factor of 2.8 might be possible. Eight pickups has been determined as the limit, because $400 \Omega$ pickups would be necessary due to the impedance change at a $\mathrm{T}$ junction type combiner, to have a $50 \Omega$ connection to the EOM. This is well above the vacuum impedance and requires tiny components. A less radical option is the combination of four pickups for a potential improvement of a factor of 2 .

Compared to both preceding pickup generations, the voltage is approximately proportional to the radius of the circular pickup surface. This may indicate a bunch-pickup ratio where only the pickup width, but not its length, is relevant.

\section{Printed circuit board BAM}

For short bunches the transition to a short rectangular pickup on a printed circuit board (PCB) with a trace thickness still larger than the bunch length appears possible. This concept possibly allows for a $100 \mathrm{GHz}$ pickup without the drawback of smaller dimensions. Further benefits are the possibility to use well-known components with precise production methods and well-described materials. The transmission lines (TLs) and combination network may be realized on the PCB reducing the rf path, which is specifically important to prevent dispersion effects in broadband quasi-TEM TLs. A microstrip is favorable for its width, but entails dispersion and is less shielded. Therefore, it is planned to use a microstrip for coupling to the field and a stripline (SL) for the combination network.

A preliminary simulation, shown in Fig. 9, of a PCB based pickup was done with a $1.55 \mathrm{~mm}$ wide and about $15 \mathrm{~mm}$ long $50 \Omega \mathrm{SL}$ in a $\epsilon_{\mathrm{r}}=4.03$ substrate disk with $10 \mathrm{~mm}$ aperture inside. For a $20 \mathrm{pC}$ bunch the simulation returns a slope of about $1270 \mathrm{mV} \mathrm{ps}^{-1}$. The SL pickup is exceeding the current as well as the $90 \mathrm{GHz}$ cone-shaped 

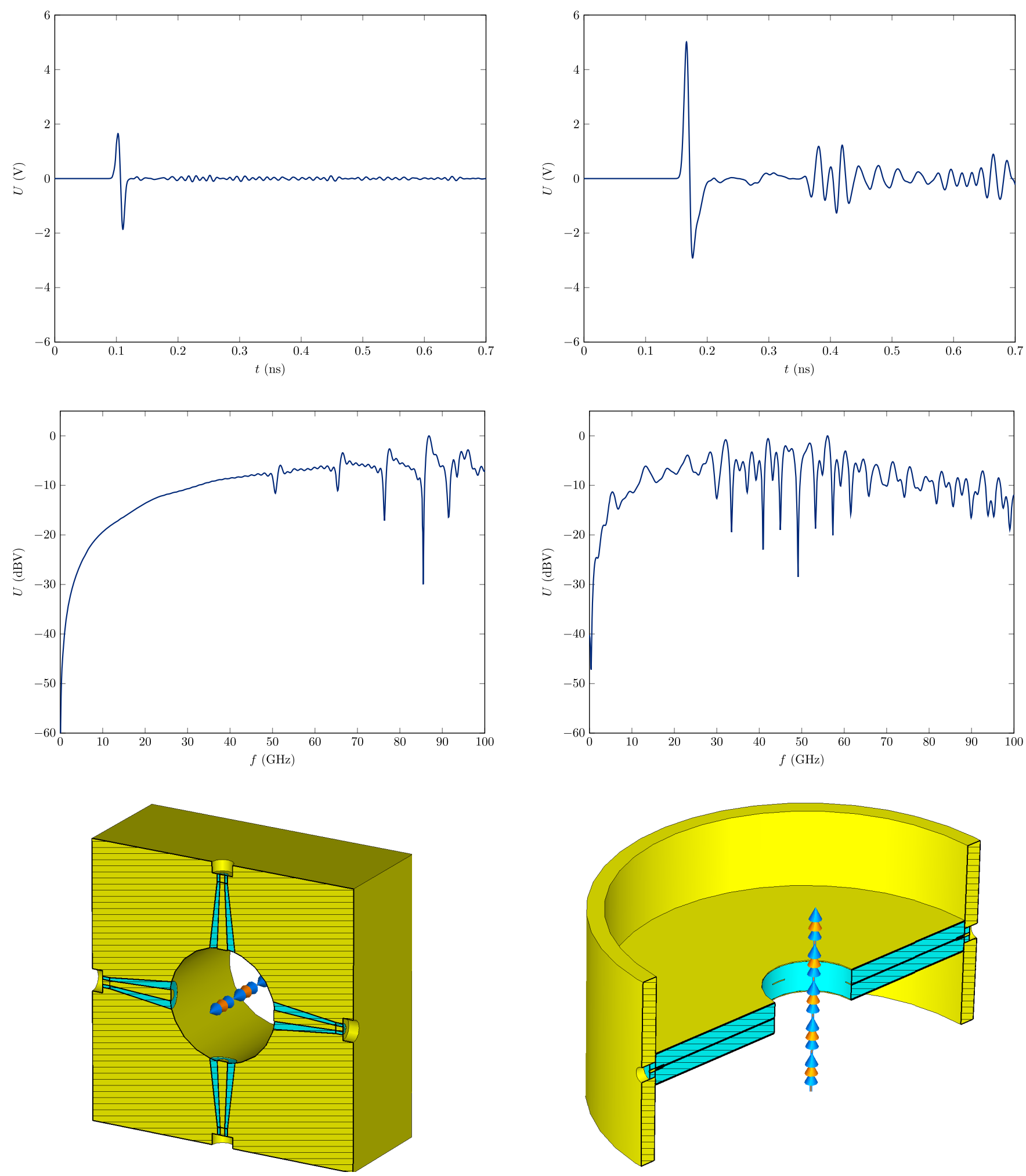

FIG. 8. The signal in time domain (top) and its normalized spectrum (center) taken at the end of the vacuum feedthrough of a single pickup as well as the simulation model with $90 \mathrm{GHz}$ coneshaped pickups (bottom), adapted from [44].

FIG. 9. The signal in time domain (top) and its normalized spectrum (center) taken at the end of the vacuum feedthrough of a single pickup as well as the simulation model with $50 \Omega$ stripline pickups (bottom), adapted from [44]. 
pickups, but does not achieve the performance of a generation 1 pickup of equal aperture. Furthermore, crosstalk and reflections at the vacuum feedthrough as well as the open pickup end generate delayed but significant ringing.

\section{CONCLUSION}

A high bandwidth cone-shaped pickup with $10 \mathrm{~mm}$ aperture leads to a significant improvement by reduction of the distance and increase of the bandwidth. If a maximum voltage is of no concern, a configuration of first generation pickups in a $10 \mathrm{~mm}$ beampipe is a simple solution estimated sufficient for bunch charges down to $4 \mathrm{pC}$. In case of ultrashort bunches a PCB-type BAM may be suited to support $100 \mathrm{GHz}$ without the drawback of reduced dimensions. With the current design restrictions, a signal combination is inevitable. Further studies of an integrated combination network are required to reduce signal reflections and losses. Furthermore, it is necessary to investigate the properties of PCB boards regarding vacuum suitability and radiation hardness. Specifically, potential damages caused by beam incidence need to be assessed.

\section{ACKNOWLEDGMENTS}

This work is supported by the German Federal Ministry of Education and Research (BMBF) under Contract No. 05K19RO1. The contribution is a version of [44], edited for publication in the Physical Review Accelerators and Beams (PRAB) Special Edition for the 9th International Beam Instrumentation Conference, IBIC 2020.

[1] E. J. Jaeschke, S. Khan, J. R. Schneider, and J. B. Hastings, Synchrotron Light Sources and Free-Electron Lasers: Accelerator Physics, Instrumentation and Science Applications, 2nd ed. (Springer International Publishing, Cham, 2020).

[2] E. A. Seddon, J. A. Clarke, D. J. Dunning, C. Masciovecchio, C. J. Milne, F. Parmigiani, D. Rugg, J. C. H. Spence, N. R. Thompson, K. Ueda, S. M. Vinko, J. S. Wark, and W. Wurth, Short-wavelength free-electron laser sources and science: A review, Rep. Prog. Phys. 80, 115901 (2017).

[3] C. M. Günther, B. Pfau, R. Mitzner, B. Siemer, S. Roling, H. Zacharias, O. Kutz, I. Rudolph, D. Schondelmaier, R. Treusch, and S. Eisebitt, Sequential femtosecond X-ray imaging, Nat. Photonics 5, 99 (2011).

[4] W. Lu, B. Friedrich, T. Noll, K. Zhou, J. Hallmann, G. Ansaldi, T. Roth, S. Serkez, G. Geloni, A. Madsen, and S. Eisebitt, Development of a hard X-ray split-and-delay line and performance simulations for two-color pump-probe experiments at the European XFEL, Rev. Sci. Instrum. 89, 063121 (2018).

[5] J. B. Rosenzweig et al., Generation of ultrashort, high brightness electron beams for single-spike SASE FEL operation, Nucl. Instrum. Methods Phys. Res., Sect. A 593, 39 (2008).

[6] S. Reiche, P. Musumeci, C. Pellegrini, and J. B. Rosenzweig, Development of ultrashort pulse, single coherent spike for SASE x-ray FELs, Nucl. Instrum. Methods Phys. Res., Sect. A 593, 45 (2008).

[7] W. Decking and T. Limberg, European XFEL post-TDR description, Technical Report No. XFEL.EU TN-2013004-01, European XFEL GmbH, Hamburg, Germany, 2013.

[8] T. Tschentscher, Layout of the X-ray systems at the European XFEL: Technical Report No. XFEL.EU TR2011-001, Deutsches Elektronen-Synchrotron, DESY, Hamburg, Germany, 2011.

[9] Y. Kot, T. Limberg, and I. Zagorodnov, Different charges in the same bunch train at the European XFEL, Technical Report No. DESY-13-215, Deutsches Elektronen-Synchrotron, DESY, Hamburg, Germany, 2013.

[10] M. Viti, M. K. Czwalinna, H. Dinter, C. Gerth, K. Przygoda, R. Rybaniec, and H. Schlarb, Recent upgrades of the bunch arrival time monitors at FLASH and European XFEL, in Proceedings of the 8th International Particle Accelerator Conference, IPAC'17 (JACoW, Geneva, Switzerland, 2017), pp. 695-698.

[11] F. Löhl, V. Arsov, M. Felber, K. Hacker, W. Jalmuzna, B. Lorbeer, F. Ludwig, K.-H. Matthiesen, H. Schlarb, B. Schmidt, P. Schmüser, S. Schulz, J. Szewinski, A. Winter, and J. Zemella, Electron Bunch Timing with Femtosecond Precision in a Superconducting Free-Electron Laser, Phys. Rev. Lett. 104, 144801 (2010).

[12] J. Hong, J.-H. Han, C. Kim, and H.-R. Yang, Bunch arrival time monitor test at PAL-XFEL ITF, in Proceedings of the 7th International Particle Accelerator Conference, IPAC2016 (JACoW, Geneva, Switzerland, 2016), pp. 223225.

[13] H.-S. Kang et al., Hard X-ray free-electron laser with femtosecond-scale timing jitter, Nat. Photonics 11, 708 (2017).

[14] T. Ohshima, S. Matsubara, H. Maesaka, and Y. Otake, Variation of beam arrival timing at SACLA, in Proceedings of the 34th International Free-Electron Laser Conference, FEL2012 (JACoW, Geneva, Switzerland, 2013), pp. 317320.

[15] J. C. Frisch et al., Femtosecond operation of the LCLS for user experiments, in Proc. 1st Int. Particle Accelerator Conf. (IPAC'10), Kyoto, Japan (JACoW Publishing, Kyoto, 2010), pp. 2287-2289, https://ref.ipac19.org/reference/ show/39223.

[16] S. Cao, Y. Leng, R. Yuan, and J. Chen, Optimization of beam arrival and flight time measurement system based on cavity monitors at the SXFEL, IEEE Trans. Nucl. Sci. 68, 2 (2021).

[17] S. Cao, J. Chen, Y. Leng, and R. Yuan, Beam arrival time measurement at SXFEL, in Proceedings of the 6th International Beam Instrumentation Conference, IBIC2017 (JACoW, Geneva, Switzerland, 2017), pp. 193-195.

[18] X. Liu, L. Hua, L. Lai, Y. Leng, R. Yuan, and N. Zhang, Electro-optic modulator based beam arrival time monitor for SXFEL, in Proceedings of the 7th International Beam Instrumentation Conference, IBIC2018 (JACoW, Geneva, Switzerland, 2018), pp. 396-399. 
[19] M. Ferianis, E. Allaria, E. Ferrari, G. Gaio, G. Penco, F. Rossi, and M. Veronese, How the optical timing system, the longitudinal diagnostics and the associated feedback systems provide femtosecond stable operation at the FERMI free electron laser, High Power Laser Sci. Eng. 4, 1 (2016).

[20] V. Arsov, M. Aiba, M. Dehler, F. Frei, S. Hunziker, M. Kaiser, A. Romann, and V. Schlott, Commissioning and results from the bunch arrival-time monitor downstream the bunch compressor at the SwissFEL injector test facility, in Proceedings of the 36th International Free Electron Laser Conference, FEL2014 (JACoW, Geneva, Switzerland, 2014), pp. 933-936.

[21] V. Arsov, P. Chevtsov, M. Dach, S. Hunziker, M. Kaiser, D. Llorente Sancho, A. Romann, V. Schlott, M. Stadler, and D. M. Treyer, First results from the bunch arrivaltime monitors at SwissFEL, in Proceedings of the 7 th International Beam Instrumentation Conference, IBIC2018 (Ref. [18]).

[22] S. Schulz, M. K. Czwalinna, M. Felber, M. Fenner, C. Gerth, T. Kozak, T. Lamb, B. Lautenschlager, F. Ludwig, U. Mavrič, J. Müller, S. Pfeiffer, H. Schlarb, C. Schmidt, C. Sydlo, M. Titberidze, and F. Zummack, Few-femtosecond facility-wide synchronization of the European XFEL, in Proceeding of the 39th International Free Electron Laser Conference, FEL'19 (JACoW, Geneva, Switzerland, 2019), pp. 318-321.

[23] A. Angelovski, A. Kuhl, M. Hansli, A. Penirschke, S. M. Schnepp, M. Bousonville, H. Schlarb, M. K. Bock, T. Weiland, and R. Jakoby, High bandwidth pickup design for bunch arrival-time monitors for free-electron laser, Phys. Rev. ST Accel. Beams 15, 112803 (2012).

[24] A. Angelovski, A. Penirschke, R. Jakoby, M. K. Czwalinna, C. Sydlo, H. Schlarb, and T. Weiland, Pickup signal improvement for high bandwidth BAMs for FLASH and European-XFEL, in Proceedings of the International Beam Instrumentation Conference, IBIC'13 (JACoW, Geneva, Switzerland, 2013), pp. 778-781.

[25] A. Penirschke, W. Ackermann, M. K. Czwalinna, M. Kuntzsch, and H. Schlarb, Concept of a novel highbandwidth arrival time monitor for very low charges as a part of the all-optical synchronization system at ELBE, in Proceedings of the 8th International Beam Instrumentation Conference, IBIC'19 (JACoW, Geneva, Switzerland, 2019), pp. 553-556.

[26] J. Kim, J. Burnham, J. Chen, F. X. Kärtner, F. Ö. Ilday, F. Ludwig, H. Schlarb, A. Winter, M. Ferianis, and D. Cheever, An integrated femtosecond timing distribution system for XFELs, in Proceedings of the 10th European Particle Accelerator Conference, EPAC'06 (JACoW, Geneva, Switzerland, 2006), pp. 2744-2746.

[27] T. Lamb, M. K. Czwalinna, M. Felber, C. Gerth, T. Kozak, J. Müller, H. Schlarb, S. Schulz, C. Sydlo, M. Titberidze, and F. Zummack, Large-scale optical synchronization system of the European XFEL with femtosecond precision, in Proceedings of the 10th International Particle Accelerator Conference, IPAC'19 (JACoW, Geneva, Switzerland, 2019), pp. 3835-3838.

[28] A. Kuhl, A. Angelovski, A. Penirschke, S. Schnepp, T. Weiland, R. Jakoby, M. K. Bock, M. Bousonville, P. Gessler,
H. Schlarb, J. Rönsch-Schulenburg, and J. Rossbach, Analysis of new pickup designs for the FLASH and XFEL bunch arrival time monitor system, in Proceeding of the 10th European Workshop on Beam Diagnostics and Instrumentation for Particle Accelerators, DIPAC'11 (JACoW, Geneva, Switzerland, 2011), pp. 125-127.

[29] M. K. Bock, M. Bousonville, M. Felber, P. Gessler, T. Lamb, S. Ruzin, H. Schlarb, B. Schmidt, and S. Schulz, Benchmarking the performance of the present bunch arrival time monitors at FLASH, in Proceedings of the 10th European Workshop on Beam Diagnostics and Instrumentation for Particle Accelerators, DIPAC'11 (Ref. [29]), pp. 365-367.

[30] Y. Yin, E. Schulte, and T. Ekelöf, Recovery of CTF beam signals from a strong wakefield background, in Proceedings of the 1995 IEEE Particle Accelerator Conference, PAC'95 (JACoW, Geneva, Switzerland, 1995), Vol. 4. pp. 2622-2624.

[31] A. Angelovski, M. K. Bock, M. Bousonville, P. Gessler, R. Jakoby, A. Kuhl, A. Penirschke, J. Rönsch-Schulenburg, J. Roßbach, H. Schlarb, S. Schnepp, and T. Weiland, Pickup design for a high resolution bunch arrival time monitor for FLASH and XFEL, in Proceedings of the 10th European Workshop on Beam Diagnostics and Instrumentation for Particle Accelerators, DIPAC'11 (Ref. [29]), pp. 122-124.

[32] V. Arsov, F. Buechi, P. Chevtsov, M. Dach, M. Heiniger, S. Hunziker, M. Kaiser, R. Kramert, A. Romann, and V. Schlott, Design and commissioning of the bunch arrival-time monitor for SwissFEL, in Proceedings of the 6th International Beam Instrumentation Conference, IBIC2017 (Ref. [17]).

[33] M. Kuntzsch, U. Lehnert, R. Schurig, J. Teichert, M. Gensch, and P. Michel, Bunch compression dependent jitter analysis with large spectral range, in Proceedings of IBIC2015 (JACoW, Geneva, Switzerland, 2015), pp. 43-45.

[34] A. Angelovski, M. Kuntzsch, M. K. Czwalinna, A. Penirschke, M. Hansli, C. Sydlo, V. Arsov, S. Hunziker, H. Schlarb, M. Gensch, V. Schlott, T. Weiland, and R. Jakoby, Evaluation of the cone-shaped pickup performance for low charge sub-10 fs arrival-time measurements at free electron laser facilities, Phys. Rev. ST Accel. Beams 18, 012801 (2015).

[35] A. Penirschke, A. Angelovski, M. Hansli, R. Jakoby, C. Sydlo, M. K. Czwalinna, M. Bousonville, H. Schlarb, A. Kuhl, S. M. Schnepp, and T. Weiland, RF front end for high bandwidth bunch arrival time monitors in free-electron lasers at DESY, in Proceedings of the 1st International Beam Instrumentation Conference, IBIC2012 (JACoW, Geneva, Switzerland, 2012), pp. 157-161.

[36] M. Kuntzsch, S. Burger, R. Schurig, and T. Weber, Long term investigation of the degradation of coaxial cables, in Proceedings of the 7th International Beam Instrumentation Conference, IBIC2018 (Ref. [18]), pp. 552-555.

[37] C. Sydlo, F. Zummack, J. Müller, M. Felber, M. K. Czwalinna, C. Gerth, H. Schlarb, and S. Jablonski, Femtosecond timing distribution at the European XFEL, in Proceedings of FEL 2015 (JACoW, Geneva, Switzerland, 2015), pp. 669-671. 
[38] K. Przygoda, L. Butkowski, M. K. Czwalinna, H. Dinter, C. Gerth, E. Janas, F. Ludwig, S. Pfeiffer, R. Rybaniec, H. Schlarb, C. Schmidt, and M. Viti, MicroTCA.4 based optical front-end readout electronics and its applications, in Proceedings of the 5th International Beam Instrumentation Conference: IBIC2016 (JACoW, Geneva, Switzerland, 2016), pp. 67-70.

[39] S. R. Smith, Beam position monitor engineering, in Proceedings of the 7th Beam Instrumentation Workshop, BIW'96, Beam Instrumentation Workshop No. 7 (AIP, New York, 1996), pp. 50-65.

[40] R. E. Shafer, Beam position monitoring, AIP Conf. Proc. 212, 26 (1990).

[41] J. Y. Huang, I. H. Yu, W. H. Hwang, M. H. Chun, and S. C. Kim, Analysis of pickup signals in the time domain and the frequency domain for the beam position monitor at the Pohang Light Source, J. Korean Phys. Soc. 48, 768 (2006).

[42] K. Küpfmüller, in Einführung in die Theoretische Elektrotechnik (Springer, Berlin, 1932), Chap. 40, pp. 268272.

[43] D. Gabor, Theory of communication. Part 1: The analysis of information, J. Inst. Elect. Eng. 93, 429 (1946).

[44] B. E. J. Scheible, A. Penirschke, M. K. Czwalinna, H. Schlarb, W. Ackermann, and H. De Gersem, Evaluation of a novel pickup concept for ultralow charged short bunches in X-ray free-electron lasers, in Proceedings of the 9th International Beam Instrumentation Conference, IBIC'20 (JACoW, Geneva, Switzerland, 2020), pp. 145149. 